\title{
Denis CHARTIER
}

Institut de Recherche pour le Développement — IRD

(2005)

\section{“ONG internationales environnementalistes et politiques forestières tropicales. L'exemple de Greenpeace en Amazonie."}

Un document produit en version numérique par Jean-Marie Tremblay, bénévole, professeur de sociologie au Cégep de Chicoutimi

Courriel: jean-marie tremblay@uqac.ca

Site web pédagogique : http://www.uqac.ca/jmt-sociologue/

Dans le cadre de: "Les classiques des sciences sociales" Une bibliothèque numérique fondée et dirigée par Jean-Marie Tremblay, professeur de sociologie au Cégep de Chicoutimi Site web: http://classiques.uqac.ca/

Une collection développée en collaboration avec la Bibliothèque Paul-Émile-Boulet de l'Université du Québec à Chicoutimi Site web: http://bibliotheque.uqac.ca/ 
Cette édition électronique a été réalisée par Jean-Marie Tremblay, bénévole, professeur de sociologie au Cégep de Chicoutimi à partir de :

Denis Chartier

Institut de Recherche pour le Développement — IRD

"ONG internationales environnementalistes et politiques forestières tropicales. L’exemple de Greenpeace en Amazonie”.

Un article publié dans la revue Anthropologie et sociétés, vol. 29, no 1, 2005, pp. 103-120. Québec : Département d'anthropologie, Université Laval, 2005, 258 pp.

Le 13 avril 2007, la direction de la revue Anthropologie et sociétés nous autorisait à diffuser tous les articles du numéro intitulé : "Forêts tropicales" dans Les Classiques des sciences sociales. Nous remercions également Érudit de nous avoir donné ses fichiers pdf-textes de tous ces articles.

g.f Courriels :

Pauline Curien, PhD $\quad$ Pauline.Curien@ant.ulaval.ca et

Anthropologie et sociétés $\quad$ Anthropologie.et.Societes@ant.ulaval.ca

l'auteur (Denis Chartier): $\quad$ denis.chartier@worldonline.fr

Polices de caractères utilisée :

Pour le texte: Times New Roman, 14 points.

Pour les citations : Times New Roman, 12 points.

Pour les notes de bas de page : Times New Roman, 12 points.

Édition électronique réalisée avec le traitement de textes Microsoft Word 2004 pour Macintosh.

Mise en page sur papier format : LETTRE (US letter), 8.5'’ x 11'’)

Édition numérique réalisée le 19 avril 2007 à Chicoutimi, Ville de Saguenay, province de Québec, Canada. 


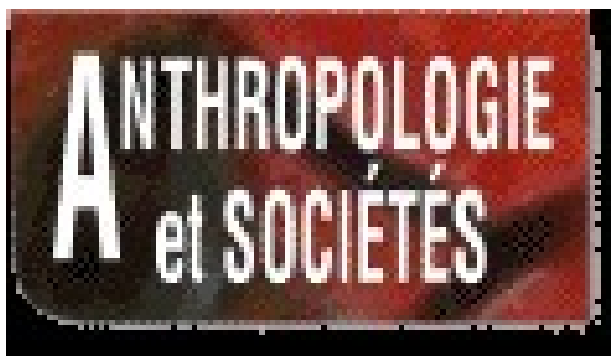

Le 13 avril 2007, la direction de la revue Anthropologie et sociétés nous autorisait à diffuser tous les articles du volume 29, no 1, intitulé : "Forêts tropicales" dans Les Classiques des sciences sociales. Nous remercions également Érudit de nous avoir donné ses fichiers pdftextes de tous ces articles.

Un grand merci à Madame Pauline Curien, Adjointe à la rédaction, pour avoir accueilli avec beaucoup d'enthousiasme notre demande de diffusion des articles sur les forêts tropicales. Merci à Madame Saillant, directrice de la revue, d'avoir répondu favorablement à notre demande de diffusion.

Je voudrais remercie Érudit pour nous avoir transmis les fichiers pdf-textes de ces articles sur les forêts tropicales, ce qui nous épargne de nombreuses heures de travail et nous permet de diffuser plus rapidement ces textes.

\section{érudit}

Courriels : $\quad$ Pauline.Curien@ant.ulaval.ca et Anthropologie.et.Societes@ant.ulaval.ca 


\section{Denis CHARTIER}

\section{"ONG internationales environnementalistes et politiques forestières tropicales. L'exemple de Greenpeace en Amazonie”.}

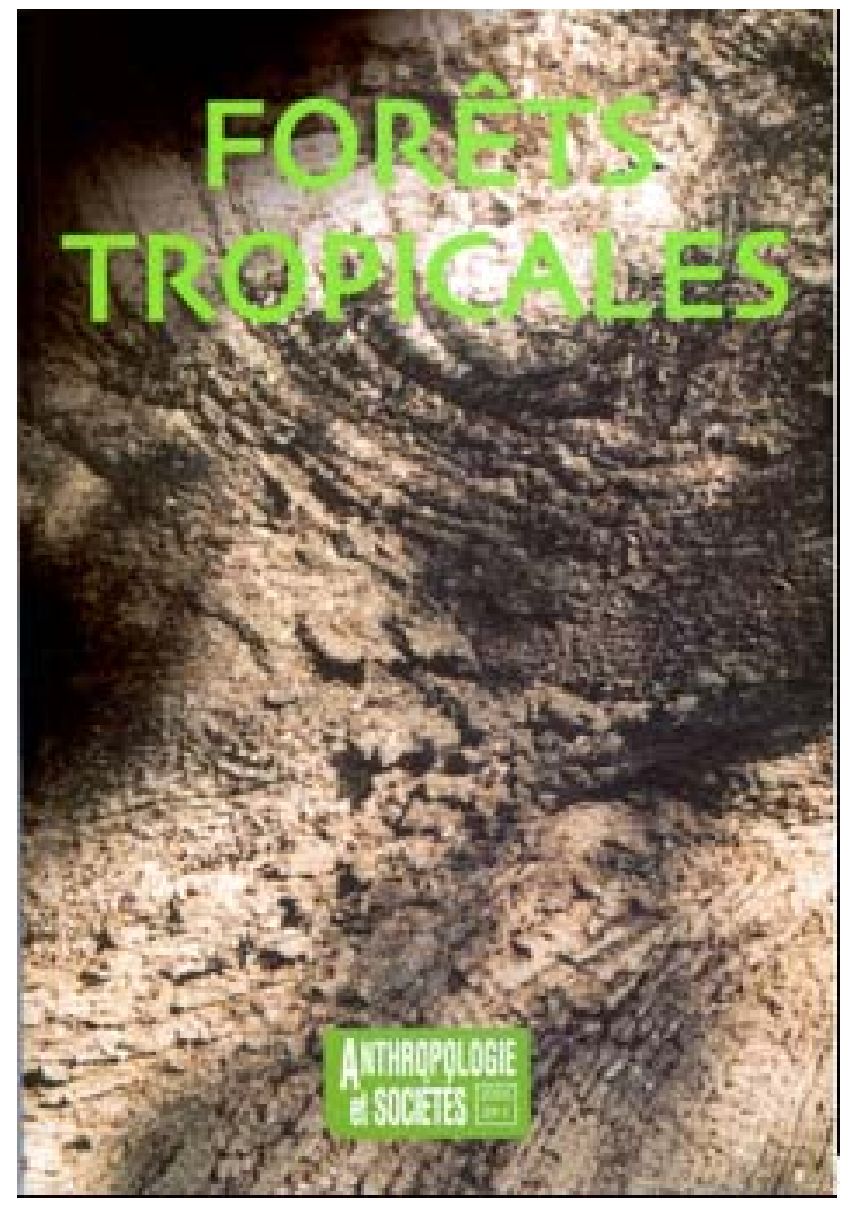

Un article publié dans la revue Anthropologie et sociétés, vol. 29, no 1, 2005, pp. 103-120. Québec: Département d'anthropologie, Université Laval, 2005, 258 pp. 


\section{Table des matières}

Introduction

Greenpeace, entrepreneur environnemental

La protection des forêts primaires: la plus importante campagne de Greenpeace

De l'efficacité des discours à la diffusion de représentations simplistes

La labellisation FSC, victoire pour la forêt amazonienne ?

Greenpeace et la mise en place de la plus grande réserve extractiviste d'Amazonie

Références

RÉSUMÉ - ABSTRACT - RESUMEN 
Denis CHARTIER

"ONG internationales environnementalistes et politiques forestières tropicales. L'exemple de Greenpeace en Amazonie”.

Un article publié dans la revue Anthropologie et sociétés, vol. 29, no 1, 2005, pp. 103-120. Québec: Département d'anthropologie, Université Laval, 2005, 258 pp.

\section{Introduction}

$\underline{\text { Retour à la table des matières }}$

De nombreux travaux montrent qu'en l'absence de politiques forestières clairement définies, les organisations non gouvernementales (ONG) se sont durablement installées « en occupant le champ du discours, laissant loin derrière non seulement les forestiers mais aussi les biologistes, les botanistes, les anthropologues, les sociologues ", etc. (Smouts 2001 : 125). En effet, ces organisations sont désormais très présentes lorsqu'il s'agit d'imposer de nouveaux concepts ou de nouvelles normes, que ce soit auprès d'organisations internationales ou d'acteurs locaux. Elles ont par exemple fait évoluer le débat sur les modes de conservation de la forêt d'une logique de préservation pure à une conception plus sensible aux dynamiques sociales locales. C'est du moins ce qui apparaît lorsque l'on consulte la Stratégie Mondiale pour la Conservation, document édité en 1980 par deux grandes ONG associées, pour l'occasion, au programme des Nations Unies pour l'environnement (UICN, WWF et PNUE 1980). C'est en effet dans ce texte à très forte portée institutionnelle que l'on aborde pour la première fois les notions de développement durable, de participation ou encore de prise en compte des pratiques des populations locales. Ce 
sont aussi des ONG internationales qui ont réussi à imposer la méthode de certification du FSC (Forest Stewardship Council) après ce que d'aucuns ont nommé une guerre des labels, guerre plus que jamais d’actualité (Arnould 1999 ; Zhouri 2002).

Cette dimension incontournable de certaines ONG, en particulier lorsqu'il s'agit de définir de nouvelles politiques forestières tropicales et internationales, appelle bon nombre de questions sur la nature et l'origine des discours qu'elles développent. Il en va de même pour les répercussions qu'elles ont sur les politiques et pratiques en matière de gestion des espaces forestiers. En effet, quelles sont les représentations véhiculées par ces organisations? Quels sont les fondements éthiques et scientifiques des usages et pratiques qu'elles défendent ? N'y a-t-il pas interférence entre les logiques internes de ces ONG et les politiques encouragées ? Et finalement, quels sont les impacts réels de leurs actions sur les politiques et les pratiques en matière de protection et de gestion des espaces forestiers ?

Nous allons apporter quelques éléments de réponse en analysant les discours et les pratiques utilisés lors des principales campagnes de Greenpeace consacrées à l'Amazonie, du milieu des années 1990 au début des années 2000. Nous nous attacherons à observer les discours afférents à la campagne de lutte contre l'exploitation clandestine du mahogany ${ }^{1}$ et visant à l'imposition d'une consommation de bois écocertifié FSC (Forest Stewardship Council). Nous analyserons les pratiques de terrain de Greenpeace par le biais de ce qu'elle a entrepris, en collaboration avec des communautés amazoniennes, qui appelaient de leurs voeux la création d'une réserve extractiviste.$^{2}$

1 Swietenia macrophylla; le mahogany est le nom commercial de l'acajou d'Amérique.

2 Les réserves extractivistes (Resex) sont des aires protégées par la loi brésilienne, destinées à la conservation et à la gestion durable des ressources naturelles dont nous reparlerons ultérieurement. 


\section{Greenpeace, entrepreneur environnemental}

Il n'est pas anodin de choisir Greenpeace afin d'illustrer l'influence des ONG dans la mise en place de nouvelles normes ou politiques forestières. Tout en restant conscient qu'il est parfois périlleux de généraliser à partir d'un cas particulier, du fait de la grande diversité qui règne sous l'appellation $\mathrm{ONG}^{3}$, nous estimons qu'une analyse s'appuyant sur cette organisation reste pertinente afin de mieux comprendre le rôle effectif de ces organisations. Par son engagement récent mais important dans la lutte pour la protection des forêts primaires et les moyens d'action substantiels dont elle dispose, Greenpeace se trouve souvent à la proue de coalitions d'ONG dans les débats internationaux ayant trait aux forêts tropicales (Zhouri 2001 ; Smouts 2001). Analyser son discours revient ainsi à observer celui de ses partenaires qui peuvent être, selon les circonstances, le WWF (World Wide Fund for Nature), FoE (Friends of the Earth) ou d'autres ONG environnementales internationales. Dans tous les cas, il s'agit toujours d'ONG parmi les plus influentes sur la scène des négociations nationales et internationales. Nous avons aussi choisi Green-

3 Comme nous avons pu le montrer dans de précédents travaux (Chartier 2002a et b), nous estimons qu'il est important de se méfier des généralisations abusives lorsque l'on parle d'ONG. Oublier la diversité qui règne sous cette appellation conduit en effet à prendre le risque de tirer des conclusions générales ou d'illustrer sa recherche en s'appuyant sur des études qui concernent des organisations radicalement différentes de celles étudiées. Pour ne donner que quelques exemples, il n'est pas rare que certaines ONG soient sous la tutelle plus ou moins directe de gouvernements alors que d'autres sont des organisations juridiquement reconnues comme ONG mais représentantes d'entreprises commerciales associées pour défendre un secteur industriel particulier. Dans les deux cas, nous sommes bien loin de «l'ONG citoyenne », indépendante, porteuse d'un nouveau rapport au monde, et il est hasardeux d'analyser ces organisations selon les mêmes procédés. 
peace parce qu'elle apparaît comme très représentative du monde des ONG internationales, que l'on considère son influence croissante ou les contradictions qu'elle doit affronter.

Plus précisément, nos précédents travaux ont montré que tout en conservant une attitude continuellement critique vis-à-vis des excès matérialistes, consuméristes et productivistes des sociétés industrielles avancées, Greenpeace s'est professionnalisée et s'est engagée dans un processus accentué de bureaucratisation, d'institutionnalisation et de centralisation (Chartier 2002). L'organisation a désormais des atours de firme multinationale. Avec un budget de près de 160 millions d'Euros (261 millions de dollars canadiens), elle contrôle 38 bureaux nationaux ${ }^{4}$, effectue des investissements directs dans plusieurs pays, et sa structure organisationnelle assure la cohésion du groupe et l'internationalisation de ses activités. La place occupée par Greenpeace dans la « société civile internationale » ainsi que sur l'échiquier politique global ne cesse ainsi de s'étendre grâce à l'amplitude de ses échelles d'intervention institutionnelle et fonctionnelle. Elle s'étend aussi grâce à l'utilisation de nombreux modes d'action ${ }^{5}$ entraînant une maîtrise affirmée de différentes métriques ${ }^{6}$. L'organisation est en effet capable d'influer sur les décisions prises par les instances internationales ou gouvernementales en pratiquant un lobbying direct et indirect. Elle a aussi les moyens de dénoncer sur le terrain des pratiques environnementales critiquables sans être gênée par des contingences spatiales (entre autre grâce à sa grande maîtrise des meilleurs

4 L’ONG disposait en 2002 de 38 bureaux nationaux ou régionaux. Elle était présente dans 41 pays et sur tous les continents à l'exception de l'Afrique (Greenpeace 2002).

5 Greenpeace utilise les modes d'action suivants : l'action militante de terrain, l'action coup de poing, le lobbying direct ou indirect, l'expertise, l'étude ou le conseil, l'action judiciaire et l'information.

6 Les métriques définissent des façons de mesurer la distance au sein d'un espace donné. En ce sens, elles ne s’appliquent pas uniquement à des distances géométriques, mais concernent au contraire une multitude de mesures de la proximité ou de l'éloignement. Parmi ces métriques, on peut opérer une distinction centrale, selon que la métrique concerne un espace continu (métrique topographique) ou un espace discontinu (métrique topologique). La première se rapporte au territoire, dans lequel une mesure donnée de la distance se retrouve en tout lieu de l'espace considéré. Les métriques topologiques sont celles des réseaux, dans lesquels les emprises sont linéaires (Lévy 1999). 
moyens de communication et grâce aux importants moyens de transport dont elle dispose). Comme beaucoup d'ONG, cette organisation est cependant confrontée à de notables contradictions. En se structurant selon les mêmes logiques que certaines multinationales pour gagner en efficacité et en tentant de s'autofinancer pour échapper au piège de la "récupération " 7 , l'organisation a dû adopter certaines valeurs du monde marchand et plus spécifiquement celles d'efficacité et de rentabilité. Afin d'assurer sa pérennité et celle de ses activités, Greenpeace est en effet contrainte d'exister médiatiquement au risque de voir le nombre de ses adhérents chuter. Ces dynamiques structurelles internes ont bien sûr des effets sur le choix des campagnes et des discours de l'ONG, puisque, en plus de son objectif premier consistant à agir sur les problèmes environnementaux, elle doit intégrer de nombreux éléments relevant plutôt du marketing afin de s'assurer de la rentabilité des discours et des campagnes engagées.

Tous ces éléments, que nous retrouvons sous des formes similaires lorsque l'on analyse les plus importantes ONG internationales environnementales (Chartier 2002), ne sont bien entendu pas sans effet sur les politiques mises en place par Greenpeace concernant les forêts tropicales.

\section{La protection des forêts primaires : la plus importante campagne de Greenpeace}

$\underline{\text { Retour à la table des matières }}$

Greenpeace a commencé à s'intéresser aux forêts primaires au début des années 1990. D’abord mineure dans le budget des dépenses par rapport aux champs d'action traditionnels de l'ONG (nucléaire et désarmement, océans et substances toxiques), la campagne forêt est vite devenue le principal champ d'action de l'organisation en termes financiers avec un budget de 4,3 millions d'Euros en 2001 (Green-

7 Les ressources de l'ONG proviennent pour 95\% de dons individuels. L'organisation refuse en effet tout financement en provenance de gouvernements, d'instances internationales ou d'entreprises privées. 
peace 2002). Dans un premier temps plutôt orienté vers la protection des forêts primaires boréales, Greenpeace a vite élargi ce champ d'action en s’intéressant aux forêts tropicales humides en général et à l'Amazonie en particulier. Même si elle a engagé quelques actions en Afrique ou en Papouasie Nouvelle-Guinée ${ }^{8}$, l'organisation a de plus en plus investi en Amazonie à partir du milieu des années 1990, pour finalement créer un bureau amazonien en 1997, géré par le siège international à Amsterdam.

Dans sa stratégie de conservation des forêts primaires, Greenpeace joue sur deux fronts. Le premier est celui des rencontres internationales où l'ONG tente de pousser les gouvernements à s'engager financièrement dans la conservation des forêts primaires, de faire cesser l'exploitation et le commerce illégal de produits issus de forêts anciennes et finalement d'engager une politique d'éco-certification des produits bois et papier utilisés dans les marchés publics ${ }^{9}$. Le deuxième front, plus visible, est celui de l'information ou des actions de terrain visant officiellement à sensibiliser et à construire des opinions publiques, afin d'influencer les principaux décideurs des politiques forestières. Nous ne parlerons ici que très sommairement du lobbying réalisé auprès des instances internationales (cela faisant l'objet de nos recherches actuelles) ; nous développerons par contre les messages et représentations délivrés par l’ONG et les répercussions qu'ils peuvent avoir sur les politiques forestières.

8 Au début des années 1990, Greenpeace s’est associée à une coalition d’ONG afin d'aider diverses communautés de Papouasie-Nouvelle-Guinée et des Îles Salomon à trouver d'autres solutions que la vente de leurs droits d'exploitation (sur des centaines de milliers d'hectares de forêts anciennes) aux entreprises d'exploitation industrielle, grâce au développement de formes d'exploitation durables du bois et des produits non ligneux.

9 Récemment, l’organisation a envoyé des délégations aux rencontres suivantes : à la VIe réunion des Parties de la Convention sur la diversité biologique (CBD) (avril 2003) pour inciter 1) à la mise en place d'un moratoire sur l'exploitation forestière dans les régions de forêts anciennes, le temps d'installer un réseau d'aires protégeant les richesses écologiques de chaque région et 2) à la création d'un fonds destiné à financer ces actions de conservation ; au Sommet du G8 en juin 2003 pour dénoncer l'exploitation illégale de bois ; à la réunion de l’OMC de Cancun (septembre 2003) pour dénoncer l'exploitation illégale du bois et faire la promotion de l'écocertificaton (Greenpeace 2003). 


\section{De l'efficacité des discours à la diffusion de représentations simplistes}

$\underline{\text { Retour à la table des matières }}$

Dans un premier temps, l'analyse des discours de sensibilisation du " public » (mais aussi des dirigeants d'entreprises ou de gouvernements) montre que l'ONG diffuse très souvent des discours manichéens mettant au grand jour les problèmes sans nécessairement chercher à prendre la mesure de leur complexité. En effet, la lecture des documents publiés par l'organisation a tout d'abord montré que le terme de forêt était à jamais défini, alors qu'aucun accord général n'existe sur sa définition. L'organisation propose par ailleurs une vision tout à fait monolithique des forêts primaires qu'elle définit comme « des forêts qui se sont établies grâce aux évènements naturels et qui sont très peu touchées par l'homme » (Greenpeace 1999 : 1). Par l'intermédiaire d'une présentation textuelle ou cartographique de ces forêts à deux instants différents, l'un après la dernière grande glaciation et l'autre à la fin du XXe siècle (Greenpeace 2003), elle opère très souvent un saut dans le temps qui exclut toute prise en considération des évolutions naturelles qu'ont connues ces espaces entre ces deux périodes. Elle propose ainsi une vision en apparence très radicale qui consiste à présenter l'homme comme le destructeur exclusif de ces milieux. Cette opposition entre l'homme et la forêt est une vision tout à fait simpliste qui résonne profondément au sein de l'opinion publique, la narrative édénique ayant ses origines dans notre propre mythe de création (Cronon 1996 ; Salter 1996). Cette vision écarte toute réflexion sur les milliers d'années de coévolution et peut même, dans certains cas, remettre sérieusement en question la perception des forêts primaires. Il est en effet acquis aujourd'hui que certains espaces amazoniens considérés encore récemment comme "vierges » sont en définitive des forêts qui ont connu une anthropisation pré-contact 
(avant l'arrivée des Européens en Amérique) ${ }^{10}$. Ne pas prendre en considération ces éléments, c'est d'une certaine façon omettre que l'homme puisse créer de la biodiversité comme certains travaux sur les peuples des forêts tropicales le montrent désormais (Balée 2000 ; Bahuchet et al. 2001). Mais c'est surtout associer, de façon simpliste, activités humaines et destruction des forêts primaires et de la biodiversité 11 .

Ces quelques éléments suffisent à montrer que les discours scientifiques publics de l'organisation ne prennent pas en compte la complexité des problèmes. Si cette attitude permet de faciliter la communication avec les adhérents et d'identifier facilement des responsables contre lesquels lutter, elle pose néanmoins un problème quant à la nature de l'opinion publique que l'on construit et aux conséquences que cela peut entraîner.

Pour des raisons stratégiques (induites par le projet même de Greenpeace, mais aussi par la nécessité de pérenniser l'ONG), l'organisation a fait le choix de mettre en place des politiques médiamétriques qui impliquent la diffusion de ces discours manichéens et excessivement pragmatiques, mettant à jour les problèmes sans prendre la mesure de leur complexité. Cela a le mérite d'être efficace quant au message à diffuser, tout en étant rentable pour l'organisation et les luttes engagées. En jouant sur l'émotion et sur le mythe moderne de la forêt vierge, l'ONG s'assure la possibilité de lever des fonds substantiels. Le problème est qu'elle participe à la construction ou au renforcement de représentations erronées qui, indirectement, peuvent avoir des conséquences sur les politiques forestières. On peut en effet estimer que ce renforcement de l'image édénique de la forêt rend difficile l'instauration de représentations plus réalistes concernant les enjeux de la conservation forestière. Du coup, des organisa-

10 Nous faisons ici référence à une étude pluridisciplinaire démontrant que des espaces forestiers guyanais considérés comme vierges sont en fait des forêts qui étaient habitées et exploitées il y a encore quelques centaines d'années (informations obtenues lors d'un entretien avec Pierre Grenand, anthropologue à l'IRD le 20 octobre 2001).

11 Il est intéressant de constater que l'organisation ne précise jamais de quelle biodiversité elle parle, ce qui ajoute un élément de plus à l'imprécision du discours. 
tions diffusant un autre discours basé sur d'autres représentations (en particulier celles qui incluent l'action anthropique) se trouvent face à de grandes difficultés lorsqu'il s'agit d'obtenir des fonds ou une audience en vue de mener une action intégrant la complexité. C'est du moins ce qui point à l'horizon lorsqu'on analyse les campagnes de Greenpeace visant à imposer un mode d'exploitation de la forêt, ces dernières ne semblant laisser que peu de place aux discours et pratiques qu'elle ne défend pas.

\section{La labellisation FSC, victoire pour la forêt amazonienne?}

$\underline{\text { Retour à la table des matières }}$

Ces discours et représentations ont fréquemment été associés à la principale campagne de l'ONG visant à démontrer que la première menace pour l'Amazonie était l'exploitation forestière commerciale (Greenpeace 1998). Trois objectifs imbriqués furent particulièrement valorisés dès le milieu des années 1990 : la lutte contre l'exploitation industrielle mais surtout illégale du bois ; la protection du mahogany ainsi que la promotion d'une consommation de bois dont l'origine serait connue et qui aurait été écocertifiée suivant des critères du Forest Stewardship Council (FSC). Afin d'atteindre ces objectifs en Amazonie, l'organisation a tout d'abord formé une coalition avec des groupes locaux brésiliens et commencé une campagne contre les entreprises du bois, l'objectif étant de combiner les actions locales avec une pression sur le marché anglais, leader mondial de la consommation de mahogany (Padua 1997). Cette campagne, qui a rapidement été étendue à toute l'Europe, a eu un impact retentissant.

Du côté brésilien, le gouvernement a publié dès 1996 un moratoire de deux ans sur les permis d'exploitation pour le mahogany et le virola en Amazonie (moratoire prolongé par la suite). Du côté européen, les campagnes de dénonciation ont tout d'abord incité le gouvernement anglais en 2000 (mais aussi français en 2002) à promettre de 
n'utiliser que des produits certifiés issus d'une gestion durable dans ses marchés publics. En 2002, après plusieurs années de pression de Greenpeace et d'autres ONG, le mahogany a aussi été protégé par son inscription en Annexe II de la CITES (Convention sur le commerce international des espèces de faune et de flore sauvages menacées d'extinction). Si ces éléments laissent entrevoir que les campagnes des ONG ont influencé les programmes politiques nationaux ou internationaux, il s'avère que l'influence des ONG sur la demande des consommateurs a aussi conduit certaines multinationales à changer de politique ${ }^{12}$.

Ces quelques exemples montrent l'ampleur du succès avec lequel Greenpeace et ses associés (WWF, FoE, etc.) ont réussi à influencer les politiques des gouvernements et des entrepreneurs économiques. Afin de mieux percevoir les conséquences de ces nouvelles politiques, penchons-nous maintenant sur les principaux postulats scientifiques qui les ont fondées.

Tout d'abord, Greenpeace appuie ses campagnes sur l'idée que l'exploitation forestière commerciale du bois (en particulier celles que mènent les compagnies transnationales) est la première cause de déforestation. Ce postulat n'est que partiellement vrai, même s'il ne fait aucun doute que les causes de cette déforestation ont évolué et que l'arrivée en masse de compagnies transnationales montre bien que la question de l'exploitation forestière est un élément clef de compréhension des processus de déforestation en Amazonie. Cependant, il est dangereux d'oublier, au risque de proposer des politiques forestières inadaptées, que « les trois quarts du déboisement enregistré dans les pays membres du traité de coopération de l'Amazonie résultent de l'expansion de terres agricoles, [et] que l'essentiel de la production

12 Le groupe Ikea, leader mondial dans le secteur de l'ameublement, s'est ainsi engagé à s’approvisionner uniquement en bois de forêts gérées durablement et certifiées par le FSC. Le groupe Lapeyre (plus grand revendeur de bois amazonien en Europe) a fait de même en 2000 en s'engageant à s’approvisionner en bois certifié FSC d'ici à 2005. Dans la même année, les deux plus grosses enseignes de bricolage françaises, Castorama et Leroy Merlin ont approché Greenpeace pour s'engager, au sein du Club d'acheteurs FSC français, à commercialiser uniquement des produits FSC d’ici 2005 (Greenpeace 2003). 
annuelle de bois matériau est destiné au marché intérieur » (Smouts $2001: 46)$.

Greenpeace prétend aussi que le label du FSC est le seul système d'éco-certification crédible et valable pour tous les types de forêts 13 . Nous n'entrerons pas ici dans un débat sur l'histoire et la validité de ce label, d'autres l'ont déjà très bien fait (Karsenty 1997 ; Arnould 1999 ; Brédif et Boudinot 2001 ; Zhouri 2002). On peut seulement noter en s'appuyant sur les auteurs cités que : 1) les normes du FSC sont des normes de performance qui s'attachent aux résultats plus qu'aux comportements, en se fondant sur des critères peu précis ; 2) que leur caractère trop général les rend difficiles à évaluer et laisse donc une grande marge d'appréciation à l’organisme accrédité ; 3) ce label favorise plutôt les grands propriétaires (capables d'amortir leurs investissements sur de vastes superficies) au détriment des petits et des moyens qui se trouvent dans l'incapacité de remplir les exigences du FSC sur leurs parcelles. Enfin, il est bon de rappeler qu'il existe de nombreuses normes mises en place par des gouvernements, des groupements d'entreprises, etc. Pour ne donner qu'un exemple, nous pouvons citer la norme de procédure ISO (International Organization for Standardisation). Affirmer qu'il n'en existe pas d'autres est donc faux et relève plus d'une bataille de marketing entre chaînes de distribution que d'une démarche scientifique visant à trouver les meilleurs moyens de gérer les forêts.

Pour finir, Greenpeace affirme qu'en n'utilisant que du bois certifié, les consommateurs européens résoudront en grande partie le problème de la déforestation en Amazonie. Ce postulat est difficilement défendable surtout lorsque l'on sait que seulement 10\% du bois brésilien va à l'exportation ; que la production de bois tropicaux ne représente que le cinquième de la production mondiale de bois ; et qu'en 2001, 90\% des forêts certifiées étaient des forêts tempérées ou boréa-

13 « En pratique, la seule façon de s’assurer que le bois vient de forêts gérées légalement et suivant des critères de gestion durable est de s’approvisionner en bois certifié FSC » (Greenpeace : 2005); «Les Principes et Critères du FSC s'appliquent à toutes les forêts de la planète, tropicales, tempérées et boréales qu'elles soient primaires, secondaires ou de plantation » (Greenpeace : 2005b). 
les. Finalement, la consommation de bois labellisé par le FSC ne représente qu'une infime partie des bois tropicaux commercialisés et des bois récoltés. Il est donc actuellement faux de proposer ce type de consommation comme solution miracle ${ }^{14}$.

La critique de ces trois postulats montre que Greenpeace construit une partie de ses propositions sur des vérités parfois discutables. Cela oblige à s'interroger sur la pertinence des propositions que fait l'organisation, surtout lorsque l'on considère à quel point elle peut influencer les politiques forestières. S'il ne fait aucun doute que les actions de l'ONG permettent de garder la question de la «bonne » gestion forestière au programme des organisations internationales, des gouvernements ou des entrepreneurs économiques, il semble en revanche que les politiques qu'elle induit ne soient pas toujours les meilleures. En effet, et contrairement à ce qui est annoncé, les vraies causes de la dégradation forestière ne seront pratiquement pas affectées par le geste d'un consommateur qui achètera du bois tropical certifié, ce commerce ne concernant qu'une infime partie du bois récolté. À cet argument s'ajoutent deux critiques exprimées par Marie-Claude Smouts (2001) qui s'appliquent à notre cas d'étude. D'une part, la recherche de certification pour des îlots de forêt au milieu de vastes zones mal gérées introduit une fragmentation de l'espace peu compatible avec une notion d'aménagement forestier. D'autre part, la fabrication de demande de produits certifiés crée une attente immédiate qui laisse croire qu'il existe des solutions simples, rapides et uniformes pour toutes les forêts du monde alors que « rien n'est plus éloigné de la forêt que cette éthique de l'urgence » (Smouts 2001 : 308). Finalement et compte tenu de l'influence effective de Greenpeace, il semble que l'organisation détourne parfois involontairement les regards, les recherches et les politiques engagées des causes primordiales de la déforestation ainsi que des « bonnes » solutions de gestion.

14 Depuis 2004, Greenpeace semble prendre la mesure de ces contradictions en proposant des campagnes qui visent les consommateurs des pays du Sud. Elle a ainsi lancé un programme appelé « cidade amiga da Amazônia » qui vise à aider les villes brésiliennes qui le désirent à créer une législation municipale éliminant le bois d'origine illégale de tous les achats municipaux (Greenpeace 2004). 
En faisant le choix du discours et des propositions manichéennes pour gagner en efficacité, l’ONG limite les débats (et les propositions) sur les solutions susceptibles de régler les problèmes de déforestation. Ce sont les logiques internes à l'organisation qui lui imposent ces méthodes aux conséquences critiquables. Greenpeace doit en effet privilégier des logiques médiamétriques pour assurer sa pérennité et continuer son action. Ces logiques entrent alors en contradiction avec le projet même de l'ONG qui, involontairement, ne contribue plus vraiment à résoudre les problèmes de déforestation. C'est du moins ce qui apparaît lorsque l'on analyse les luttes de l'ONG concernant l'exploitation commerciale des forêts tropicales. Par contre, l'organisation semble développer d'autres méthodes et pratiques lorsqu'il s'agit de la conservation des forêts tropicales.

\section{Greenpeace et la mise en place de la plus grande réserve extractiviste d'Amazonie}

$\underline{\text { Retour à la table des matières }}$

Hormis les luttes évoquées ci-dessus, Greenpeace appelle de ses voeux la délimitation de tous les territoires indigènes, la promotion de toutes les formes d'extraction durable des ressources forestières et l'augmentation du nombre de réserves extractivistes consacrées à l'exploitation de latex et d'autres produits non ligneux. C'est donc logiquement (et un peu paradoxalement au regard des campagnes de communication citées) que deux des principales actions de terrain de l'ONG, depuis 1999, ont consisté à travailler, d'une part avec les indiens deni de la vallée du Purus- Amazonas (participation à la délimitation du territoire de ces derniers), d'autre part avec les populations caboclos du município (commune) de Porto de Moz. Nous ne parlerons ici que de la dernière action qui a consisté à soutenir des communautés désireuses de promouvoir leur projet de réserve extractiviste. 
Porto de Moz est situé sur le bas Xingu, dans l'État brésilien du Pará ${ }^{15}$. Ce município, constitué en grande partie de terres inondables (várzeas), est assez représentatif de l'occupation traditionnelle de l'Amazonie, avec plus d'une centaine de communautés vivant principalement d'agriculture familiale, de pêche et de produits de la forêt. Depuis quelques années, il est aussi devenu représentatif des atteintes aux droits de l'homme et à l'environnement, fréquents sur de nombreux fronts pionniers amazoniens.

Tout d'abord, des bateaux de pêche à fort tonnage (geleiras) sont arrivés dans la région à la fin des années 1980, pour mener des campagnes de pêche industrielle qui ont conduit à la diminution des réserves halieutiques. Durant la même période, l'exploitation forestière a considérablement augmenté. Compte tenu de l'épuisement des ressources sur des centres de production plus anciens et de l'implantation de scieries dans le município, l'exploitation est en effet devenue très intense, d'autant plus que le maire de Porto de Moz est le propriétaire de deux des trois plus importantes scieries de la région. Cette exploitation de la forêt a été facilitée par la situation foncière du município qui reste relativement floue. Si la plupart des terres appartiennent à l'État fédéral, rien n'empêche les éleveurs (fazendeiros) ou les exploitants de bois (madereiros) de profiter de l'absence de titres légaux de propriété pour s'approprier de grandes superficies de façon illégale. Face à ces appropriations, le droit à la terre des petits paysans s'est trouvé fragilisé alors qu'il était jusque là garanti, même si ces derniers ne possédaient pas de titre officiel (Salgado et al. 2002).

Face à l'épuisement des ressources naturelles halieutiques ou forestières 16 et à la naissance d'importants conflits d'intérêts, en particulier entre madereiros et communautés caboclas, les organisations locales (l'Église, les associations de pêcheurs et de producteurs ru-

15 D’une superficie équivalant à la moitié du territoire belge (17 $000 \mathrm{~km} 2)$, il abrite une population de près de 25000 habitants répartis entre le centre urbain (6927 habitants) et les communautés caboclas de l’intérieur.

16 L'opposition de certaines communautés à l'avancée de la pêche industrielle a été le premier acte de résistance organisé. Il a été lancé et largement soutenu par les prêtres catholiques du município, tous adhérents aux préceptes de la théologie de la libération. 
raux, le syndicat des travailleurs ruraux) ont entamé une série de discussions internes dans le but de changer cet état de fait. Elles ont ainsi organisé, avec l'appui du LAET (Laboratoire agroécologique de la transamazonienne), un diagnostic centré sur l'exploitation du bois et la pêche. Lors d'un séminaire de restitution portant sur l'état des ressources naturelles, un plan d'action a été défini pour organiser les communautés, résoudre leurs problèmes fonciers, former techniquement les petits agriculteurs (en particulier à l'utilisation et à la commercialisation de bois) et organiser une surveillance de l'état des ressources naturelles. Après la création d'un Comité des ressources naturelles, constitué par les organisations locales afin d'agir au niveau des organismes publics, mais aussi au sein des communautés, les dirigeants de ces communautés ont estimé que la reconnaissance de leurs droits traditionnels sur des « terres communautaires » représentait une bonne stratégie de contrôle de l'avancée des exploitants forestiers, tout en garantissant la terre aux descendants des familles ${ }^{17}$. Il est cependant très vite apparu qu'il existait un problème de reconnaissance et de législation concernant ces aires communautaires, et que cette stratégie était difficile à mettre en place pour plus de 100 communautés différentes. Il est également apparu que ces délimitations (qui ne concernaient chaque fois qu'une petite superficie allant de 3000 à 13 $000 \mathrm{ha}$ ) ne suffisaient pas pour protéger le reste du territoire. L'option de la réserve extractiviste (Resex) s'est alors présentée, peu avant l'arrivée de Greenpeace.

Le concept de Resex a surgi au milieu des années 1980 comme une solution pour les travailleurs du caoutchouc du Brésil - résultat de la lutte menée par Chico Mendes, le leader des seringueiros de l'Acre (un des 27 États du Brésil) assassiné en 1987. Il s’agissait alors de créer des zones réservées à l'exploitation des produits de la forêt sur

17 La création de huit aires communautaires a permis d'engager au sein des communautés des discussions sur une utilisation durable des ressources naturelles, la formation ou la réorganisation d'associations communautaires, la démarcation des aires protégées par l'ouverture de layons à la machette, l'exécution de relevés de leur potentiel, l'identification des formes d'usage existantes et la mise en place de formations à la planification de l'usage des ressources forestières. À ce sujet, on peut lire l'ouvrage Vivre avec la forêt, qui retrace bien les différentes étapes et les atouts de la mise en place de ces aires protégées (Castellanet et al. 2002). 
lesquelles les seringueiros pourraient vivre et travailler sans craindre l'expulsion et l'abattage des arbres. Le concept a fait fortune et la Resex a été reconnue en 1989 par le gouvernement brésilien comme un instrument de consolidation des politiques publiques environnementales. La réserve extractiviste a été la première unité de conservation conciliant deux concepts longtemps perçus comme dissociés: la conservation et l'exploitation. Rompant avec des conceptions préservationnistes, cette méthode de conservation garantit aux familles qui y vivent un droit d'usage sur leurs terres. Celles-ci peuvent donc continuer à vivre de leurs pratiques et activités économiques traditionnelles tout en participant à la conservation des ressources naturelles (Benatti 2002 : 296).

Lorsque l'on confronte le concept de réserve extractiviste aux discours de Greenpeace précédemment évoqués, il peut paraître surprenant que l'ONG ait choisi de soutenir le projet de ces communautés. Pourtant, l'organisation a clairement aidé ces populations afin que leur demande soit entendue par le gouvernement. Le premier contact important entre les membres de Greenpeace et les populations locales de Porto de Moz a été réalisé par Marcello Marquesini (l'un des responsables de Greenpeace-Brésil) à la fin des années 1990. Dans le cadre d'une enquête visant à réaliser une carte de l'exploitation illégale du bois dans la région du bas Xingu (Pará-Brésil) ${ }^{18}$, ce dernier a rencontré quelques responsables des organisations locales et s'est ainsi aperçu que les communautés du município avaient des revendications très précises. Après avoir consulté un certain nombre de documents du Syndicat des travailleurs ruraux, Marquesini a proposé aux communautés locales de les aider à obtenir leur réserve nommée Verde para sempre (vert pour toujours) ${ }^{19}$. Cette collaboration s'est concrétisée par une action « coup de poing » de retentissement national et international orchestrée par Greenpeace. Du 19 au 21 septembre 2002,

18 Marcello Marquesini est aujourd'hui membre du gouvernement de Lula et directeur de la surveillance des forêts pour l'Ibama (Institut brésilien de l'environnement et ressources renouvelables).

19 Ces informations ont été obtenues lors d'interviews réalisées auprès de Claudio Wilson Barbosa (coordinateur du comité de développement durable de Porto de Moz) et Idalino Numes de Assis (responsables du syndicat des travailleurs ruraux) - Porto de Moz, mai 2003. 
l'organisation, accompagnée d'environ 500 personnes des différentes communautés, a créé un barrage de bateaux sur la rivière Jaraucu afin de protester contre la destruction de la forêt amazonienne et pour la création de la Resex. L'action fut un énorme succès puisque deux barges de bois illégal, propriétés du frère du maire du município (principal exploitant de bois de la région), furent arraisonnées non sans échauffourées très médiatisées. Au terme de l'action, le bilan a été perçu comme très positif par tous les acteurs en présence, c'est du moins ce qui se dégage de nos entretiens.

L'action de Greenpeace auprès de ces populations caboclas a clairement montré que l'ONG a apporté une aide certaine à ces communautés pour communiquer et défendre leur projet en toute sûreté. Il est en effet indéniable que le maire du município aurait usé plus volontiers de la force pour faire taire les résistants si Greenpeace n’avait pas été présente. Il est aussi certain que ce projet de réserve a reçu une attention nationale et internationale. Une émission spéciale sur une chaîne nationale brésilienne a été consacrée à cette action et aux problèmes de ces communautés et, parallèlement, Greenpeace a largement utilisé cette action pour divulguer ses activités en Amazonie. Inversement, ces communautés ont permis à Greenpeace de faire passer dans la presse des informations sur l'exploitation industrielle du bois (officielle et clandestine) ainsi que sur certaines pratiques de conservation en mettant en valeur son association avec les populations locales. Tous ces éléments montrent un autre visage de l'action de Greenpeace. Pourtant, si le fait d'aider ces populations marque une volonté d'inciter à la mise en place de politiques de conservation socio-environnementales intégrant une certaine complexité, des problèmes apparaissent lorsque l'on confronte les discours et les pratiques aux réalités de terrain.

Dans tous les documents de l'ONG se référant à cette action, les communautés caboclas du município de Porto de Moz sont présentées comme les «héritiers de Chico Mendes », comme des populations traditionnelles vivant en harmonie avec leur milieu naturel (Greenpeace 2002b). L'image que diffuse Greenpeace au sujet de ces populations est donc clairement celle d'une solution humaine optimale pour une exploitation douce d'une Amazonie postulée comme indispensable à la survie de l'humanité. Pourtant, si nous avons vu qu'il 
existe pour ces communautés un réel souci d'organisation afin de préserver les ressources naturelles, il est périlleux de mythifier à ce point leur culture. Nous avons en effet pu constater sur le terrain que la " vocation écologique " de ces communautés est parfois récente, très inégale et pas toujours ancrée dans les pratiques. Nous avons par exemple observé que dans l'une des communautés les plus dynamiques du point de vue de la lutte pour la Resex (São João do Cupari), l'achat récent de quelques bufflonnes a conduit très rapidement à une croissance exponentielle des troupeaux, induisant des problèmes de salubrité de l'eau potable pendant la saison sèche. Nous avons aussi été frappé de voir que bon nombre d'animaux ou d'espèces végétales n’étaient pas nommées ou connues par ces populations, ce qui confirmerait certaines études selon lesquelles les savoirs des caboclos sur la faune et la flore locales seraient bien inférieurs aux savoirs amérindiens dont ils sont issus. Il ne resterait en effet que des acquis pratiques dont seraient écartés « les éléments superflus en termes de survie et improductifs en termes de rentabilisation immédiate " (Grenand et Grenand 1990 : 31). Finalement, et au-delà de ces atténuations qui ne doivent surtout pas minimiser la réelle volonté de ces populations de préserver le milieu naturel, ces caboclos qui ne luttaient jusqu'à récemment que pour préserver quelques terres à exploiter se sont livrés à une opération de transcodage (selon l'expression de Lascoumes [1994]) traduisant les objectifs qui étaient les leurs dans un registre utilisé par les écologistes étrangers ${ }^{20}$. Ce transcodage n’est pas nécessairement problématique si l'on sait qu'il existe. Il l'est beaucoup plus lorsque, comme Greenpeace, on soutient ces populations et leurs pratiques sans jamais avoir réalisé une étude de terrain et sans conscience explicite des différences fondamentales qui caractérisent les représentations en présence 21 . Pour ces populations, la forêt amazonienne est avant tout une ressource et un lieu de subsistance où le travail est dur, alors que pour Greenpeace, c'est un espace naturel emblème d'une nature perdue. Il en va de même pour la réserve extractiviste perçue par Greenpeace comme une solution optimale de préservation de la forêt alors qu'elle est pour ces populations un moyen de sortir de

20 Il s’agit dans un premier temps des membres du LAET puis de ceux de Greenpeace et des quelques autres ONG présentes dans la région.

21 C'est du moins ce qui se dégage de la lecture des documents disponibles et de notre enquête de terrain réalisée en mai 2003. 
l'isolement et, par la génération de revenus, un moyen d'intégrer la société de consommation. À terme, ces différentes représentations peuvent devenir problématiques puisqu'elles montrent que ces acteurs environnementaux ont des priorités divergentes. Il est pourtant connu que la sortie de l'isolement de ces communautés, lors de la mise en place de réserves extractivistes, peut accentuer l'exploitation des ressources naturelles typiques des fronts pionniers (Lena 2002). En s'engageant auprès de ces populations, Greenpeace montre une réelle ouverture à des politiques socio-environnementales novatrices ; néanmoins, il apparaît qu'elle aide ces populations sans prendre la mesure de la complexité qui y règne, au risque de créer des effets pervers.

En conclusion, nous retiendrons que le rôle de Greenpeace est ambigu, que l'on s'intéresse aux représentations véhiculées, aux politiques proposées ou aux actions engagées. D’un côté, en étant l'un des principaux promoteurs de nouveaux concepts, de nouvelles normes et de nouvelles politiques forestières, l'organisation contribue à maintenir à l'ordre du jour la question de la bonne gestion des forêts tropicales. Elle réussit aussi à apporter une aide conséquente à certaines populations locales en difficulté, qu'il s'agisse des indiens deni ou des populations caboclas de Porto de Moz. De l'autre, l'ONG fait trop souvent l'impasse sur la prise en considération de la complexité des enjeux de la conservation forestière, au nom de logiques internes d'efficacité et de rentabilité. L'objet forêt, devenu central pour Greenpeace, la conduit à engager et porter, souvent trop rapidement, un certain nombre de normes et de représentations n’intégrant pas la complexité inhérente à ces milieux naturels. Il apparaît aussi que l'évolution des aspirations individuelles et collectives à l'égard des forêts, ainsi que les nouvelles demandes sociales apparues dans les pays du Nord et portées par les ONG, entrent souvent en contradiction avec celles des pays du Sud. C'est ce dernier point qui est le plus problématique. En effet, sans une meilleure prise en compte des relations spécifiques qui peuvent exister entre certaines populations du Sud et la nature, sans une meilleure compréhension entre le Nord et le Sud, aucune solution viable ne sera trouvée pour préserver et valoriser les forêts tropicales humides. 
Il faut donc résoudre ces problèmes de relation, d'articulation et de collaboration afin que puisse se produire un renouveau du politique rendu nécessaire par les processus de mondialisation contemporains. Cela n’a pas échappé aux responsables de Greenpeace international. De récentes entrevues nous ont en effet permis de constater qu'il existait, en plus d'une forte conscience des contradictions, une réflexion interne visant à améliorer et repenser la campagne forêt en fonction des éléments cités ici, cela valant particulièrement pour les actions amazoniennes marquées par le contexte brésilien ${ }^{22}$. La revitalisation récente de l'expression démocratique au Brésil et l'attention internationale pour l'Amazonie ont stimulé les initiatives liées au développement durable et renforcé la présence de ces nouveaux acteurs que sont les ONG (Buclet 2002). Cela a conduit à une explosion de leur nombre, synonyme de grande diversité. Que l'on s’intéresse à la taille, à la structure interne, aux sources de financement, aux champs et modes d'action, aux fondements idéologiques, aux liens avec les secteurs publics et privés, etc., cette hétérogénéité fait de ce monde des organisations non gouvernementales un espace apparemment désorganisé difficile à appréhender. Des études récentes (Buclet 2004 ; Scholz 2004) montrent pourtant qu'au-delà de cette diversité qui complexifie l'analyse, les ONG d'Amazonie brésilienne tentent d'articuler et de relier les différentes échelles, les différents acteurs et les différentes stratégies d'action. Au final, en mettant en scène des intérêts identitaires locaux, des enjeux économiques, politiques ou environnementaux nationaux et internationaux, l'Amazonie est le théâtre d'une expérimentation politique très riche où les ONG peuvent apparaître, malgré leurs contradictions et leur diversité, comme les acteurs d'un renouveau du politique indispensable à la résolution des problèmes environnementaux et sociaux contemporains.

22 Entrevues avec Shane Rattenbury (Directeur des programmes océans et forêts) et Bruno Rebelle (Directeur des programmes internationaux), réalisées à Amsterdam, au siège de Greenpeace International, les 8 et 9 décembre 2004. 


\section{Références}

$\underline{\text { Retour à la table des matières }}$

ARNOULD P., 1999, "L'écocertification ou la guerre des labels vers une nouvelle géopolitique forestière ? ", Annales de géographie, 609 : 567-582.

BAHUCHET S., P. DE MARET, F. GRENAND et P. GRENAND, 2001, Des forêts et des hommes. Bruxelles, APFT - ULB.

BALÉE W., 2000, «Qui a planté le décor en Amazonie ? », La Recherche, $333: 18-23$.

BENATTI J. H., 2002, « Formas de acesso à terra e a preservação da floresta amazônica : uma análise jurídica da regularização fundiária das terras dos quilombolas e seringueiros » : 292-297, in J. P. R. Capobianco (dir.), Biodiversidade na Amazônia Brasileira. São Paulo, Estação Liberdade / ISA.

BRÉDIF H. et P. BOUDINOT, 2001, Quelles forêts pour demain? Paris, L’Harmattan.

BUCLET B., 2002, "Les expérimentations des ONG en Amazonie : quel pouvoir pour quelle responsabilité ? », Lusotopie, $1: 263$ 282.

—, 2004, Le marché international de la solidarité. Les ONG en Amazonie brésilienne. Thèse de doctorat en sociologie politique. Paris, École des Hautes Études en Sciences Sociales.

CHARTIER D., 2002a, Le rôle de Greenpeace et du WWF dans la résolution des problèmes environnementaux. Quel espace politique pour quelles ONG ? Thèse de géographie. Orléans, Université d'Orléans. 
—, 2002b, « ONG et société civile. Un inventaire à la Prévert », Écologie et politique, 25 : 101-106.

CRONON W. (dir.), 1996, Uncommon Ground. Rethinking the Human Place in Nature. Londres, W. W. Norton \& Company. FSC, 2003, www.fsc.org

GREENPEACE, 1999, Acheter la destruction. Amsterdam, Greenpeace international.

_, 2002, Annual Report 2002. Amsterdam, Greenpeace international.

—, 2002b, Saisie de bois illégal pendant l'action de blocage des héritiers de Chico Mendes. Consulté sur Internet

(http ://www.greenpeace.org/france_fr/news), le 15 juin 2003.

—, 2003, Forêts. Consulté sur Internet

(http ://www.greenpeace.org/france_fr/campaigns/intro ?campaign _id=65718), le 13 juillet 2003.

—, 2004, Cidade amiga da Amazônia. Consulté sur Internet (http ://www.greenpeace.org.br/cidadeamiga/), le 29 mai 2004.

—, 2005a, Campagne forêts : les solutions. Consulté sur Internet (http ://www.greenpeace.org/france_fr/campaigns/intro ?campaign _id=65771), le 7 mars 2005.

—, 2005b, Écocertification FSC. Consulté sur Internet

(http ://www.greenpeace.org/france fr/extra ?campaign\%5fid=657 71\&forward\%5fsource\%5fanchor=Ecocertification\%20FSC\&item\%5

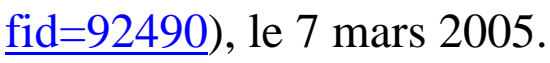

GRENAND F. et P. GRENAND, 1990, «L'identité insaisissable. Les Caboblos amazoniens », Études rurales, octobre-décembre, 120 : 17-39. 
KARSENTY A., 1997, "Certification et gestion durable des forêts : entre commerce et recherche ", Bois et forêts des tropiques, 251, $1: 76$.

LASCOUMES P., 1994, L’éco-pouvoir. Paris, La Découverte.

LENA P., 2002, "As políticas de desenvolvimento sustentável para a Amazônia : problemas e contradições », Boletim Rede Amazônica, 1, $1: 9-22$.

LEVY J., 1999, Le tournant géographique. Paris, Belin.

MOREIRA E. S., J. HÉBETTE et W. LEIÃO, 2002, « Occupation de l'Amazonie brésilienne, décentralisation et gestion des ressources naturelles : un panorama historique » : 15-23, in C. Castellanet , L. Granchamp Florentino, C. Mégevand et S. Moncorps (dir.), Vivre avec la forêt. Paris, Gret et UICN.

PADUA J. A., 1997, « La force de l'écologisme », Page deux, janvier-février : 62-72.

SALGADO I., M. CUNHA DOS SANTOS, M. AMARAL et M. MUCHAGATA, 2002, "Gestion forestière communautaire : perspectives et limites pour la conservation productive des ressources boisées en Amazonie brésilienne » : 45-53, in C. Castellanet, L. Granchamp Florentino, C. Mégevand et S. Moncorps (dir.), Vivre avec la forêt. Paris, Gret et UICN.

SCHOLZ I., 2004, Negotiating Solutions for Local Sustainable Development and the Prevention of Deforestation in the Brazilian Amazon. Bonn, German Development Institute.

SLATER C., 1996, "Amazonia as Edenic Narrative » : 114-131, in W. Cronon (dir.), Uncommon Ground. Rethinking the Human Place in Nature. Londres, W. W. Norton \& Company.

SMOUTS M. C., 2001, Forêts tropicales, jungle internationale. Les revers d'une écopolitique mondiale. Paris, Presses de Sciences Po. 
WWF, PNUE et UICN, 1980, Stratégie mondiale de la conservation. La conservation des ressources vivantes au service du développement durable. Gland, UICN-PNUE-WWF.

ZHOURI A., 2001, "Transnational Campaigns for the Amazon. NGO strategies, Trade and Official Responses », Ambiente \& Sociedade, 6-7 : 31-62.

ZHOURI A., 2002, "Amazônia : Vocação Florestal ? Notas sobre a insustentabilidade de uma visão mercadológica global », I Reunião da ANPPAS, Indaiatuba, 6-9 de Novembro.

\section{RÉSUMÉ - ABSTRACT - RESUMEN}

\section{ONG internationales environnementalistes et politiques forestières tropicales. L'exemple de Greenpeace en Amazonie}

L'analyse critique de la campagne de Greenpeace consacrée à la protection de l'Amazonie, entre le milieu des années 1990 et le début des années 2000, révèle l'impact contradictoire des ONG internationales sur les politiques forestières tropicales. D'un côté, l'ONG participe avec succès au maintien sur les agendas nationaux et internationaux de la question de la bonne gestion des forêts tropicales. Elle réussit aussi à apporter une aide conséquente à certaines populations locales, tout en étant l'un des principaux promoteurs de nouveaux concepts, de nouvelles normes et de nouvelles politiques. De l'autre, certaines logiques internes entraînent des choix stratégiques qui conduisent Greenpeace à engager et porter des normes et des représentations n’intégrant pas toujours la complexité inhérente aux milieux forestiers tropicaux. Cela peut amenuiser les débats visant à mettre à jour les meilleurs modes de conservation et de gestion de ces espaces forestiers. Ces dynamiques peuvent aussi produire les effets inverses de ceux qui sont escomptés. 
Mots clefs : Chartier, Amazonie, forêts tropicales, ONG, Greenpeace, conservation de la nature

\section{International Environmentalist NGOs and Tropical Forest Policies. The Case of Greenpeace in Amazonia}

A critical analysis of Greenpeace's conservation campaign in Amazonia, from the mid 1990s to today reveals the contradictory impact of international NGOs on tropical forest policies. On one hand, Greenpeace succeeds in maintaining the question of tropical forest management on national and international political agendas. The organisation also contributes by providing a substantial aid to local populations, whilst being at the forefront of new concepts and norms in local participation and promoting new policies. On the other hand, internal logics induce strategic decision-making which, at times, leads Greenpeace to adopt norms and representations that do not always adequately represent the complexity inherent to tropical forest environments. This in turn can restrict debates concerning appropriate conservation modalities and the management of forest spaces. Such dynamics can also produce contrary effects to those desired.

Key words : Chartier, Amazonia, tropical forests, NGOs, Greenpeace, nature conservation

\section{ONG internacionales ambientalistas y políticas forestales tropicales. El ejemplo de Greenpeace en Amazonía}

El análisis de la campaña de Greenpeace consagrada a la protección de la Amazonía, entre mediados de los años 1990 y principios de los años 2000, muestra el impacto contradictorio de las ONG internacionales sobre las políticas forestales tropicales. Por un lado, la ONG participa con éxito al mantenimiento de la cuestión de la buena gestión de los bosques tropicales en las agendas nacionales e internacionales. Esta organización logra asimismo aportar la ayuda requerida por ciertas poblaciones locales, sin perder su rol de promotor de nuevos conceptos, nuevas normas y nuevas políticas. Por otra parte, ciertas dinámicas internas inducen decisiones estratégicas que conducen a 
Greenpeace a comprometerse y promover normas y representaciones que no siempre integran la complejidad inherente a los medios forestales tropicales. Eso puede simplificar los debates que tratan de actualizar los mejores modos de conservación y de gestión de los espacios forestales. Estas dinámicas pueden asimismo producir efectos contrarios a lo que se buscan.

Palabras clave: Chartier, Amazonía, bosques tropicales, ONG, Greenpeace, conservación de la naturaleza

\section{Denis Chartier}

Institut de Recherche pour le Développement - IRD

5 rue du Carbone

45072 Orléans cedex 2

France

denis.chartier@worldonline.fr

Fin du texte 